\title{
Colonization of healthy children by Moraxella catarrhalis is characterized by genotype heterogeneity, virulence gene diversity and co-colonization with Haemophilus influenzae
}

\begin{abstract}
Correspondence
Suzanne J. C. Verhaegh

s.verhaegh@erasmusmc.nl
\end{abstract}

Received 23 June 2010

Revised 3 September 2010

Accepted 10 September 2010
Suzanne J. C. Verhaegh, ${ }^{1,2}$ Martine L. Snippe, ${ }^{1}$ Foster Levy, ${ }^{3}$ Henri A. Verbrugh, ${ }^{1}$ Vincent W. V. Jaddoe, ${ }^{2,4,5}$ Albert Hofman, ${ }^{2,5}$ Henriëtte A. Moll, ${ }^{4}$ Alex van Belkum ${ }^{1}$ and John P. Hays ${ }^{1}$

\author{
${ }^{1}$ Department of Medical Microbiology and Infectious Diseases, Erasmus MC, Rotterdam, \\ The Netherlands \\ ${ }^{2}$ The Generation R Study Group, Erasmus MC, Rotterdam, The Netherlands \\ ${ }^{3}$ Department of Biological Sciences, East Tennessee State University, Johnson City, TN, \\ USA \\ ${ }^{4}$ Department of Pediatrics, Erasmus MC, Rotterdam, The Netherlands \\ ${ }^{5}$ Department of Epidemiology, Erasmus MC, Rotterdam, The Netherlands
}

\section{INTRODUCTION}

Moraxella catarrhalis is part of the normal bacterial flora in the nasopharynx of children, although over the past two decades, it has emerged as a significant bacterial pathogen

Abbreviations: AOM, acute otitis media; CEACAM, carcinoembryonic antigen-related cell adhesion molecule; LOS, lipooligosaccharide; MLST, multilocus sequence typing; OMP, outer-membrane protein; OR, odds ratio; OMV, outer-membrane vesicle; URTI, upper respiratory tract infection. and not simply a commensal colonizer (Verduin et al., 2002). Studies have shown that the bacterium rapidly colonizes the nasopharynx soon after birth and that the carriage rate of $M$. catarrhalis in healthy children varies between 7 and $36 \%$ (Verhaegh et al., 2010). However, in children with upper respiratory tract infections (URTI), including acute otitis media $(\mathrm{AOM})$, the carriage rate increases to approximately $50 \%$ (Berner et al., 1996; Konno et al., 2006; Pettigrew et al., 2008). Otitis media itself is a particularly important URTI during early 
childhood and the primary reason for children to visit a physician (Freid et al., 1998; Plasschaert et al., 2006). Furthermore, in many countries, AOM is the most common reason for prescribing antibiotics (Gonzales et al., 2001; Leibovitz, 2003; Pichichero, 2000; Plasschaert et al., 2006) or for undergoing surgery (for the placement of grommets) (Vlastarakos et al., 2007). The most common bacterial species cultured from the nasopharynx of children during otitis media episodes are Streptococcus pneumoniae, Haemophilus influenzae, M. catarrhalis and Staphylococcus aureus, either as single pathogens or as co-cultures (Berner et al., 1996; Pettigrew et al., 2008), with the patterns of nasopharyngeal colonization by micro-organisms being important determinants for otitis media disease (Faden et al., 1991, 1997; Murphy \& Parameswaran, 2009).

With respect to M. catarrhalis, it has been shown that many factors affect nasopharyngeal carriage of this human-specific pathogen, including, for example, the presence of siblings, day care attendance and respiratory illness (Faden et al., 1997; Hendley et al., 2005; Peerbooms et al., 2002; Principi et al., 1999; Verhaegh et al., 2010). Furthermore, there is increasing information regarding the biological mechanisms facilitating M. catarrhalis-mediated colonization and disease development, with most publications stressing the importance of bacterial adherence as an essential first step in this process. To date, several important $M$. catarrhalis adhesins have been described, including the ubiquitous surface proteins UspA1 and UspA2, and the haemagglutinin/ Moraxella IgD-binding protein Hag/MID (Bullard et al., 2005; Helminen et al., 1994). UspA1 and UspA2 acquire their adherence characteristics through multifunctional binding sites, which include domains that have the ability to attach to epithelial cells via cell-associated fibronectin, laminin and vitronectin (Tan et al., 2006b). Furthermore, UspA1 includes a critical binding site for carcinoembryonic antigen-related cell adhesion molecules, which are expressed in various human tissues including respiratory epithelia (Hill \& Virji, 2003; Slevogt et al., 2008). The Moraxella IgDbinding protein, also referred to as Hag (human erythrocyte agglutinin), is also important for host attachment. MID is an outer-membrane protein (OMP) with specific affinity for soluble and cell-bound human IgD and is the only $\operatorname{IgD}$ binding protein in Moraxella (Forsgren et al., 2001).

Several other virulence-associated genes have been identified that may be associated with colonization and infection of M. catarrhalis, including the OMPs CopB, OMPCD, McaP, MhaB, MhaC, Msp22, Msp75, Msp78 and lipooligosaccharide (LOS) (Akimana \& Lafontaine, 2007; Balder et al., 2007; Lipski et al., 2007; Liu et al., 2007; Riesbeck et al., 2006; Ruckdeschel et al., 2008, 2009; Tan et al., 2006a). Furthermore, several of these virulence genes have also been associated with the induction of the humoral immune response, making them potential vaccine candidate genes. However, relatively little is known about the frequency and the extent of genotypic variation within these virulence genes, and in particular how this variation may be associated with colonization over time.
The aim of this study was to investigate the seasonal and yearly prevalence patterns of longitudinal colonization, genotypic variation and variation in virulence-associated genes of M. catarrhalis isolates colonizing children within a single and distinct geographical region. Using these data, we hoped to discover whether distinct isolate genotypes and/or identical putative vaccine candidate gene types circulated during specific time periods in healthy colonized children. We also investigated the longitudinal prevalence of $M$. catarrhalis and $H$. influenzae within our cohort of children, looking for co-colonization trends within this age group. These results will further help our understanding of $M$. catarrhalis population dynamics and provide insights into the chronological diversity and functionality of virulence-associated genes for this bacterial pathogen.

\section{METHODS}

Study population. This study was embedded within the Generation R Focus cohort, part of a population-based prospective cohort study from fetal life until young adulthood based in Rotterdam, The Netherlands (The Generation R Study) (Jaddoe et al., 2007, 2008). Within the Generation R Focus cohort, detailed assessments of fetal and postnatal growth and development were performed on 1232 pregnant Dutch women. The mothers gave birth to 1244 infants between February 2003 and August 2005, in Rotterdam, The Netherlands, of whom 138 were excluded from the study analysis because consent was withdrawn after birth. Twins were also excluded from the analysis because of genetic relatedness, leaving a total of 1079 infants. The medical ethics committee of the Erasmus Medical Center approved the study. Written informed consent was obtained from the parents of all the participants.

Infants visited the Generation R Focus cohort research centre at 1.5, 6, 14 and 24 months after birth, resulting in 630 swabs being taken at 1.5 months, 787 swabs at 6 months, 717 swabs at 14 months and 617 swabs at 24 months between November 2003 and September 2007. From 623 children, at least three swabs were available for bacterial culturing.

Bacterial isolates. Bacterial isolates were cultured from nasal swabs taken from infants at 1.5, 6, 14 and 24 months of age by a trained research nurse, using a sterile transport swab immersed in Amies transport medium. Swabs were cultured within $6 \mathrm{~h}$ of sampling, using blood agar plates containing 5\% sheep blood, chocolate agar and Haemophilus-selective agar at an incubation temperature of $35{ }^{\circ} \mathrm{C}$ in $5 \% \mathrm{CO}_{2}$ for $48 \mathrm{~h}$. Plates were examined daily for the growth of $M$. catarrhalis and/or $H$. influenzae.

Genotyping. Genotyping of M. catarrhalis isolates was performed using PFGE and multilocus sequence typing (MLST). PFGE was also performed on $H$. influenzae isolates.

PFGE for M. catarrhalis was performed as detailed by Verduin et al. (2000). Briefly, M. catarrhalis plug digestions were performed using SpeI at $20 \mathrm{U}$ per reaction and an electrophoresis protocol comprising a first block with a constant voltage of $6 \mathrm{~V} \mathrm{~cm}^{-1}$ and a pulse time from 3.5 to $25 \mathrm{~s}$ during the first $12 \mathrm{~h}$, followed by a second block of $8 \mathrm{~h}$ where the pulse time increased linearly from 1 to $5 \mathrm{~s}$. The PFGE protocol for $H$. influenzae was adapted from the work of Moor et al. (1999). Digestions were performed using SmaI at $30 \mathrm{U}$ per reaction and an electrophoresis protocol comprising a first block with a constant voltage of $6 \mathrm{~V} \mathrm{~cm}^{-1}$ and a pulse time from 6 to $8 \mathrm{~s}$ during the first $7 \mathrm{~h}$, followed by a second block of $17 \mathrm{~h}$ where the pulse time 
increased linearly from 1 to $38 \mathrm{~s}$. All PFGE patterns were analysed using BioNumerics (Applied Maths), with gel lanes normalized against a lambda DNA ladder (Bio-Rad) and band tolerance set at $1.5 \%$. PFGE products between 48.5 and $339.5 \mathrm{~kb}$ for $M$. catarrhalis and 48.5 and $485 \mathrm{~kb}$ for $H$. influenzae were included in the band matching analysis.

MLST genotyping was performed to determine whether M. catarrhalis genotypes circulating in the Rotterdam area were similar to $M$. catarrhalis genotypes worldwide and to establish genetic relationships with other internationally recognized clones available in the $M$. catarrhalis MLST database. MLST was performed on $12 \mathrm{M}$. catarrhalis isolates that had been cultured serially on three separate occasions from four children. Briefly, PCR was performed to detect and sequence the $a b c Z, a d k$, efp, fum $C, g l y R S, m u t Y$, ppa and $\operatorname{trp} E$ genes as described in the guidelines available at the MLST website (http://mlst. ucc.ie/). A touchdown thermocycling programme was used for $g l y R S$ and $a d k$. The touchdown protocol used an initial annealing temperature of $70{ }^{\circ} \mathrm{C}$, which was reduced by $1{ }^{\circ} \mathrm{C}$ per cycle over 15 PCR cycles. The following 20 cycles of amplification used an annealing temperature of $55^{\circ} \mathrm{C}$. For $p p a, e f p, a b c Z$ and $\operatorname{trpE}$, a standard PCR protocol comprising an annealing temperature of $52{ }^{\circ} \mathrm{C}$ and an elongation time of $2 \mathrm{~min}$ at $72{ }^{\circ} \mathrm{C}$ for 25 cycles was used, whilst for fumC and $m u t Y$ an annealing temperature of $55^{\circ} \mathrm{C}$ was used. PCR primer pairs were used for amplicon sequencing, except for the genes glyRS, fum $C$ and $m u t Y$, which required separate sequencing primers (primers are available at http://mlst.ucc.ie/). Allelic profiles were defined and compared with the MLST types of isolates from different geographical regions available at http://mlst.ucc.ie/.

PCR screening of virulence genes. $M$. catarrhalis isolates were grown from the glycerol stock overnight at $37^{\circ} \mathrm{C}$ on blood agar plates. DNA was extracted using the MagNA Pure LC System (Roche Applied Science). PCR was performed to detect the uspA1, uspA2, uspA2H, hag/mid and ompJ genes and 16S rRNA and LOS types as described previously (Verhaegh et al., 2008). A touchdown thermocycling programme was used for all PCRs except for hag/mid and LOS PCRs. The touchdown protocol used an initial annealing temperature of $70{ }^{\circ} \mathrm{C}$, which was reduced by $1{ }^{\circ} \mathrm{C}$ per cycle over 15 cycles of PCR. The following 20 cycles of amplification used an annealing temperature of $55{ }^{\circ} \mathrm{C}$. All isolates negative for both uspA2 and $u s p A 2 H$ genes were tested using primers uspA2end.f and uspA2end (Verhaegh et al., 2008), which amplify a conserved region found at the 3 '-end of both uspA2 and uspA2H genes.

For the hag/mid gene PCR, a standard PCR protocol comprising an annealing temperature of $55{ }^{\circ} \mathrm{C}$ and an extension time of $8 \mathrm{~min}$ at $68{ }^{\circ} \mathrm{C}$ for 25 cycles was used. The LOS-typing PCR protocol was performed as described by Edwards et al. (2005).

After amplification, 16S rRNA PCR products were digested using the enzymes FspBI $(10 \mathrm{U})$ and HhaI $(10 \mathrm{U})$ according to Verhaegh et al. (2008), to identify the 16S rRNA types (Verhaegh et al., 2008).

Virulence gene variation. Virulence gene variation was assessed using PCR-RFLP typing on three major $M$. catarrhalis virulence genes. These genes comprised the ubiquitous surface protein genes $\mathrm{A} 1$ and $\mathrm{A} 2$ (uspA1 and uspA2), and the haemagglutinin/IgD-binding protein gene hag/mid. PCR products of these genes were digested using the restriction enzyme HaeIII ( $B s u$ RI), using $10 \mathrm{U}$ per reaction mix, and incubated overnight at $37^{\circ} \mathrm{C}$. The HaeIII restriction enzyme was chosen because its recognition site(s) is found within immunologically important regions of the uspA1, uspA2 and hag/mid genes.

PCR-RFLP patterns were analysed using BioNumerics (Applied Maths), with gel lanes normalized against a $1 \mathrm{~kb}$ (uspA2 and hag/mid) and $100 \mathrm{bp}$ (uspA1) ladder and band tolerance set at $1.5 \%$.
Data analysis. To evaluate potential predictors of colonization, logistic regression analysis was performed with general estimating equations using the logit link function as implemented in the GENMOD procedure in SAS version v9.1.3 (SAS Institute, 2002, SAS 9.1.3.). Separate analyses were conducted for the binary dependent variables of presence or absence of either M. catarrhalis or $H$. influenzae in a repeated measures design. The repeated variable was children, each of whom had 1-4 swabs in the study period. Predictor variables were the presence/absence of the non-target species, year and season. Seasons were defined as winter (December-February), spring (March-May), summer (June-August) and autumn (September-November). An independent correlation matrix structure among predictor variables was used but there were no differences in assessment of significances using exchangeable or unstructured correlation matrices. Two- and three-way interaction terms were not included in the final model because (i) all covariances between model variables in all analyses were low $(r<0.04)$, (ii) exploratory analyses that excluded the repeated design showed interaction terms that did not contribute to the efficiency of the models and (iii) all, except one, of the 64 potential interaction terms for each target species were not significant.

\section{RESULTS}

\section{Bacterial isolates}

A total of $709 \mathrm{M}$. catarrhalis isolates and $621 \mathrm{H}$. influenzae isolates were cultured from a total of 2751 nasal swabs obtained during the 3-year study period. The ages of the subjects when the cultures were taken are given in Table 1.

\section{Population dynamics}

Approximate, but regular, 6-monthly cycles of peak colonization prevalence were observed over the 3-year study period for $M$. catarrhalis, with a less regular pattern observed for $H$. influenzae. Peak seasonal prevalence differed between $M$. catarrhalis and $H$. influenzae, with $M$. catarrhalis prevalence highest in autumn and winter [odds ratio $(\mathrm{OR})=1.3-2.0$ compared with spring or summer with $P<0.03$ for each comparison] and $H$. influenzae prevalence highest in winter and spring $(\mathrm{OR}=1.5-1.9 ; P<0.01)$. Each species seasonal peak represented a significantly higher risk of carriage compared with non-peak seasons and the odds of a child being colonized by one of the two pathogens were significantly increased if

Table 1. Number of $M$. catarrhalis and $H$. influenzae cultures used in this study

Total number in study population $=2751$.

\begin{tabular}{|lcc|}
\hline Age at collection (months) & M. catarrhalis & H. influenzae \\
\hline 1.5 & 73 & 44 \\
6 & 240 & 194 \\
14 & 212 & 227 \\
24 & 184 & 156 \\
& $n=709$ & $n=621$ \\
\hline
\end{tabular}


the other species was present $(\mathrm{OR}=1.5 ; P<0.0001)$. The peak in joint occurrences occurred in autumn. If the product of the individual species occurrence proportions is considered an indicator of the expected proportion of joint occurrences, chi-squared analyses showed significant excesses over expectations of joint occurrences for spring $(P<0.01)$, summer $(P<0.01)$ and autumn $(P=0.04)$. Both pathogens were at or near their peak prevalence in 2006 and so the joint prevalence was highest that year at $10.8 \%$. Conversely, the combined absence of both species in children was at its lowest in 2006 at $49.3 \%$. For $M$. catarrhalis, there were significantly higher risks in 2006 and 2007 compared with 2004 and 2005 ( $\mathrm{OR}=1.5-2.1$; $P<0.01)$. In contrast, the risk for $H$. influenzae was highest in 2006 compared with other years $(\mathrm{OR}=1.3-1.7 ; P<0.03)$ (Fig. 1).

\section{Genetic diversity of $M$. catarrhalis and $H$. influenzae isolates}

A selection of 112 isolates were arbitrarily chosen for PFGE genotyping from the $709 \mathrm{M}$. catarrhalis isolates cultured in this study, including isolates from children colonized only once during the study period and serial isolates cultured from the same child. A high degree of genotypic heterogeneity was maintained over the entire study period. While $16 \mathrm{~S}$ rRNA types 2 and 3 (lineage 2) composed a distinct clade, heterogeneity of all markers was independent of year, season and serial colonization status (Fig. 2).
MLST genotyping of $12 \mathrm{M}$. catarrhalis isolates was performed in order to determine the global context of colonizing isolates obtained from Rotterdam, The Netherlands. These 12 isolates originated from four children that were colonized with $M$. catarrhalis on three separate occasions, and represented different PFGE genotypic clusters. The Rotterdam isolates tested were found to be non-clonal in nature with many ( 8 out of 12 ) singleton MLST types, further indicating the genetic heterogeneity of $M$. catarrhalis isolates, even within a relatively restricted geographical region (Fig. 3).

Due to the significant association between $M$. catarrhalis and $H$. influenzae colonization, $36 \mathrm{H}$. influenzae isolates (including both co-colonizing and single-species isolations) were chosen for PFGE genotyping to study their genetic diversity. No single $H$. influenzae genotype or clonal cluster was associated with co-colonization events (Fig. 4).

\section{PCR screening of virulence genes}

PCR screening of the same $112 \mathrm{M}$. catarrhalis isolates for which PFGE was performed revealed an incidence of $97 \%$ (109/112) for uspA1, $80 \%$ (90/112) for hag/mid, $83 \%$ (85/ 102) for uspA2, $17 \%$ (17/102) for uspA2H and $100 \%$ (112/ 112) for ompJ. Ten isolates were found to be PCR-negative using previously published uspA2- and $u s p A 2 H$-specific primers, but positive using primers designed to amplify a conserved region of both uspA2 and uspA2H. These 10 isolates were not included in subsequent $u s p A 2$ and $u s p A 2 H$ prevalence calculations, though their inclusion as

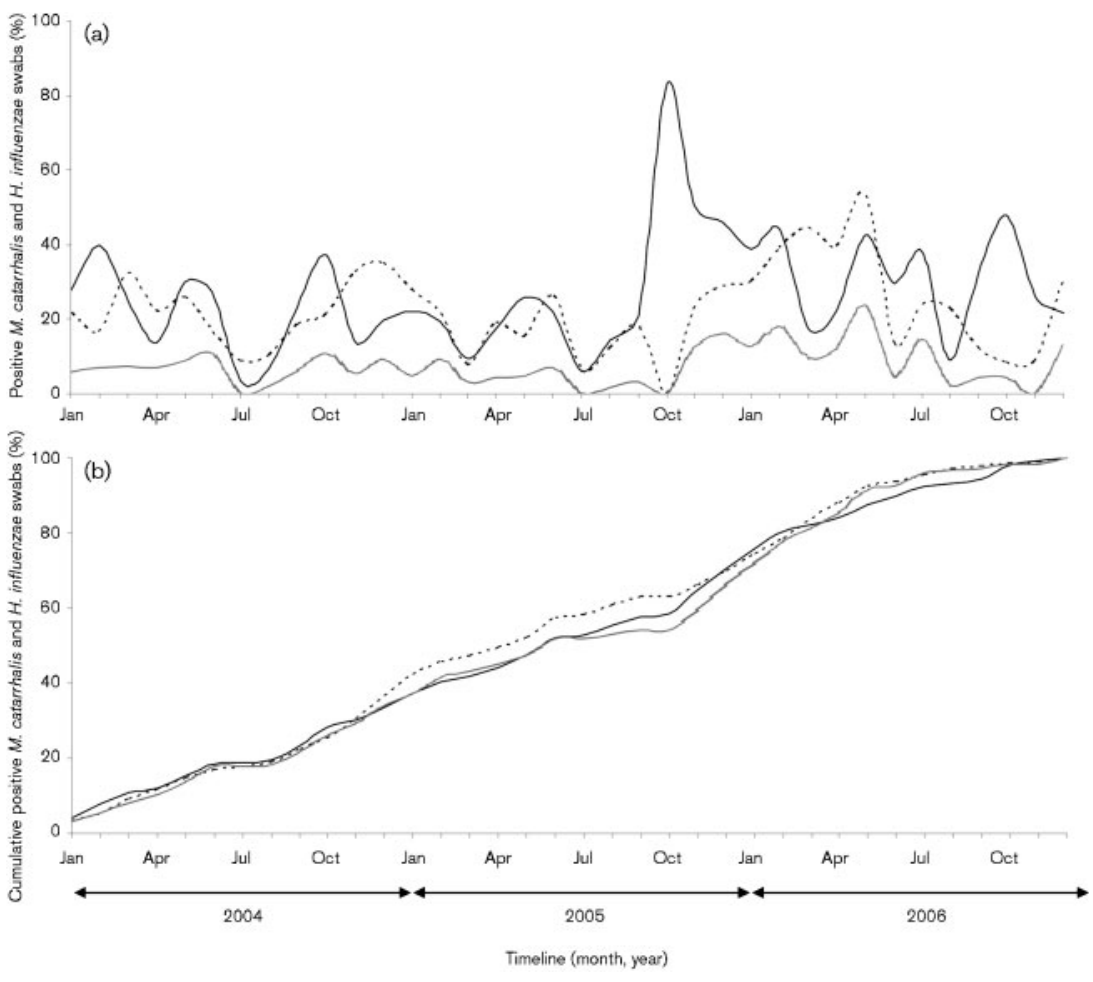

Fig. 1. Colonization prevalence of $M$. catarrhalis and $H$. influenzae during 2004-2006. (a) Proportion of $M$. catarrhalis (black line) and $H$. influenzae (dashed line) compared with the total number of swabs per month. The proportion of the joint occurrence of the two pathogens in each month compared with the total number of swabs taken per month is also shown (grey line). Note the approximate 6monthly colonization cycle (from peak to peak), especially for $M$. catarrhalis. (b) Cumulative percentage of $M$. catarrhalis-positive (black line) and $H$. influenzae-positive (dashed line) swabs recorded during the 3-year study period. Co-culture-positive swabs are also shown (grey line). The cumulative colonization burden in the study population increased at a constant rate both for pathogens and for $\mathrm{co}^{-}$ colonization over the 3-year study period. 


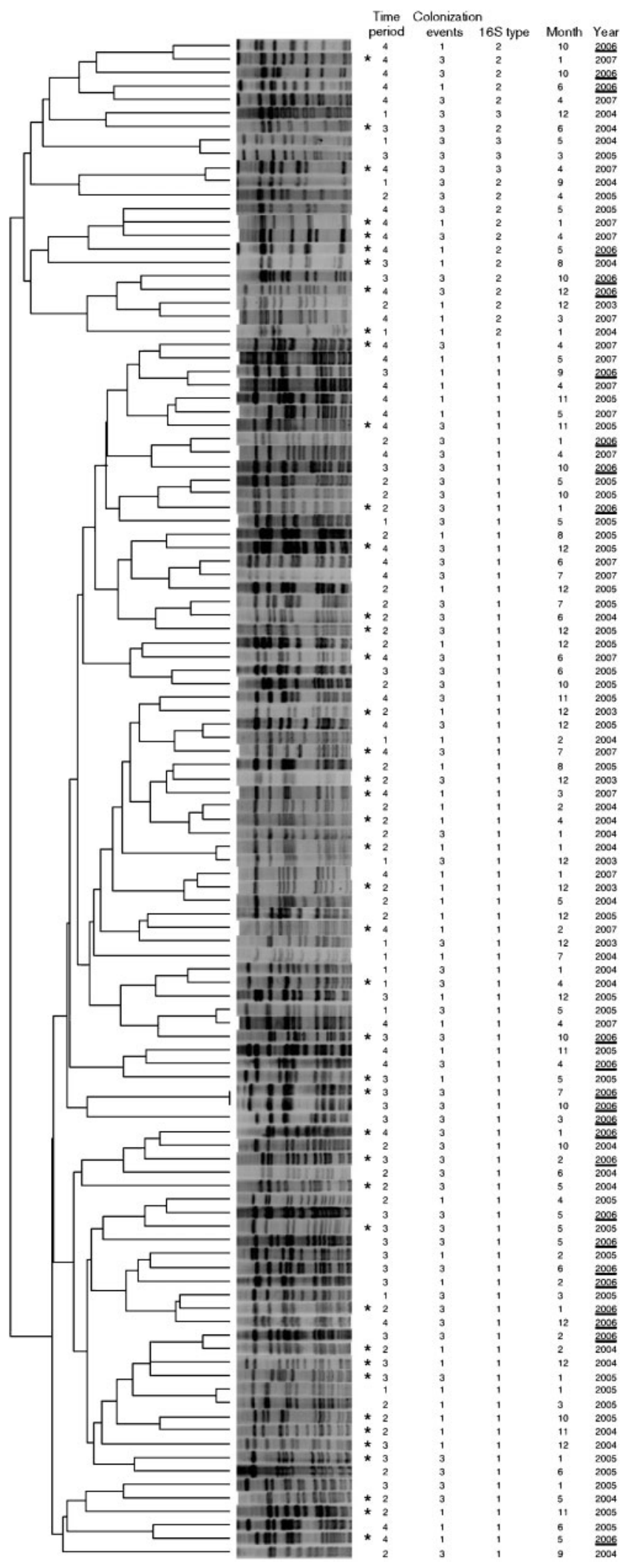

Fig. 2. Dendrogram based on PFGE genotypic variation of 112 colonizing $M$. catarrhalis isolates obtained from 74 children aged 1.5-24 months between November 2003 and September 2007 in Rotterdam. Time period after birth when nasal swabs were taken:
1, 6 weeks; 2, 6 months; 3, 14 months; 4,24 months. Colonization events: 1 , children colonized in a single sampling period only during the course of the study; 3 , children colonized three times during the study period. $16 \mathrm{~S}$ rRNA type: 1 , lineage $1 ; 2 / 3$, lineage 2 . The month and year of isolate culture from the nose of children are also shown (year of peak prevalence underlined). Cocolonization with $H$. influenzae is indicated with asterisks $\left(^{*}\right)$. No clustering of serial isolates cultured from the same child, or cocolonizing (with $\mathrm{H}$. influenzae) isolates, was observed.

either uspA2 or uspA2H would not have affected the significance of the results (Table 2).

$16 \mathrm{~S}$ rRNA gene type analysis revealed an incidence of $80 \%$ for $16 \mathrm{~S}$ rRNA type 1 (lineage 1 ), and a combined $20 \%$ for types 2 and 3 (lineage 2). All isolates that belonged to lineage 1 were associated with the presence of the hag/mid gene and OmpJ type 2.

\section{Virulence gene diversity}

PCR-RFLP was performed on amplified PCR products of the hag/mid, uspA2 and uspA1 virulence genes of $86 \mathrm{M}$. catarrhalis isolates to assess virulence gene diversity of colonizing M. catarrhalis. Fig. 5 shows the diverse nature of the PCR-RFLP patterns obtained for hag/mid, uspA2 and uspA1 circulating between 2004 and 2006 in healthy children in Rotterdam, The Netherlands. No distinct clustering of PCR-RFLP patterns was observed during particular months or years, suggesting that many different virulence gene variants circulate within colonizing $M$. catarrhalis populations at any given time. This indicates that individual gene variation is as extensive as wholegenome polymorphism.

\section{DISCUSSION}

The colonization dynamics of $M$. catarrhalis and $H$. influenzae were studied in a population of 1079 healthy children living in Rotterdam, The Netherlands. Over the 3year study period, a trend towards a regular 6-monthly $M$. catarrhalis colonization cycle (peak to peak) was observed for M. catarrhalis (which was less evident for $H$. influenzae), without a pronounced seasonal peak for either. This observation tends to contrast with other studies reporting a seasonal influence on $M$. catarrhalis colonization, although these studies also reported that higher detection rates of $M$. catarrhalis during the colder months could be a side effect of nasal hyper-secretion induced by viral illness or cold weather (Hendley et al., 2005). Marchisio et al. (2001) concluded that the seasonal influence (comparison between spring and autumn) on nasopharyngeal carriage of respiratory tract pathogens in healthy children was negligible (Marchisio et al., 2001). However, their study was limited in describing seasonal variations due to lack of observations in other years, which would have allowed a better understanding of the dynamics 


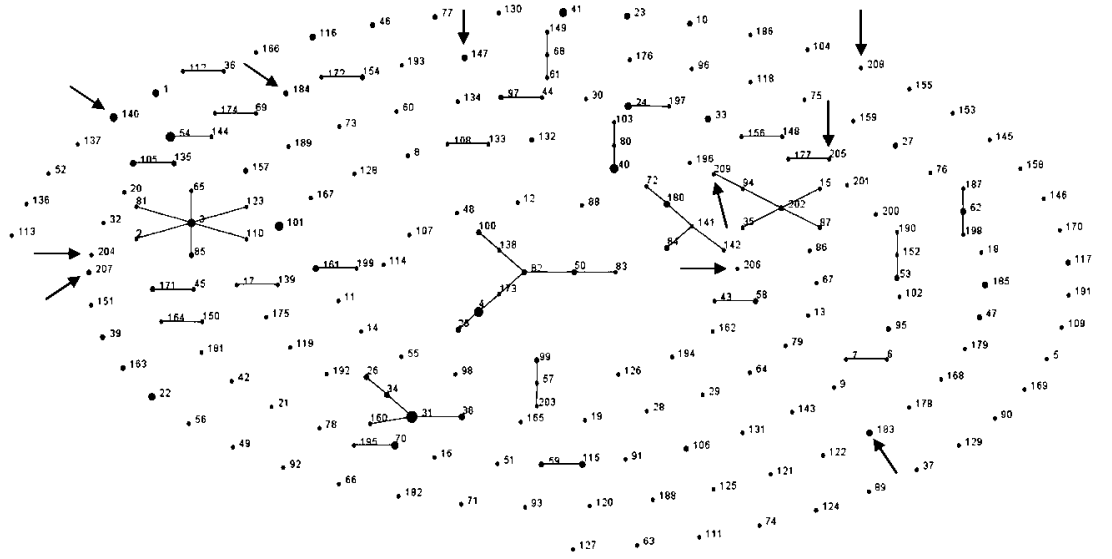

Fig. 3. MLST genotyping of $12 \mathrm{M}$. catarrhalis isolates. The sequence types assigned to the isolates are indicated with black arrows, showing the non-clonal nature of the isolates. Each dot represents a distinct MLST type, and MLST types connected with a black line possess similar allelic profiles, possibly derived from a common ancestor. of the individual pathogens. Our results suggest that colonization with $M$. catarrhalis (and to a lesser extent $H$. influenzae) occurs via regular cycles of colonization and clearance, with the apparent increase in M. catarrhalis and $H$. influenzae detection during the winter months probably being a consequence of increased viral respiratory infections, e.g. influenza, resulting in increased opportunity for secondary bacterial infection by bacterial pathogens. The link between seasonal virus infections and resultant secondary bacterial infections has been indicated by several publications (Bakaletz, 1995; Pettigrew et al., 2008; Ruuskanen et al., 1991). However, reports by Meijer et al. (2007) and Arkema et al. (2008), who collected clinical and virological data via the European Influenza

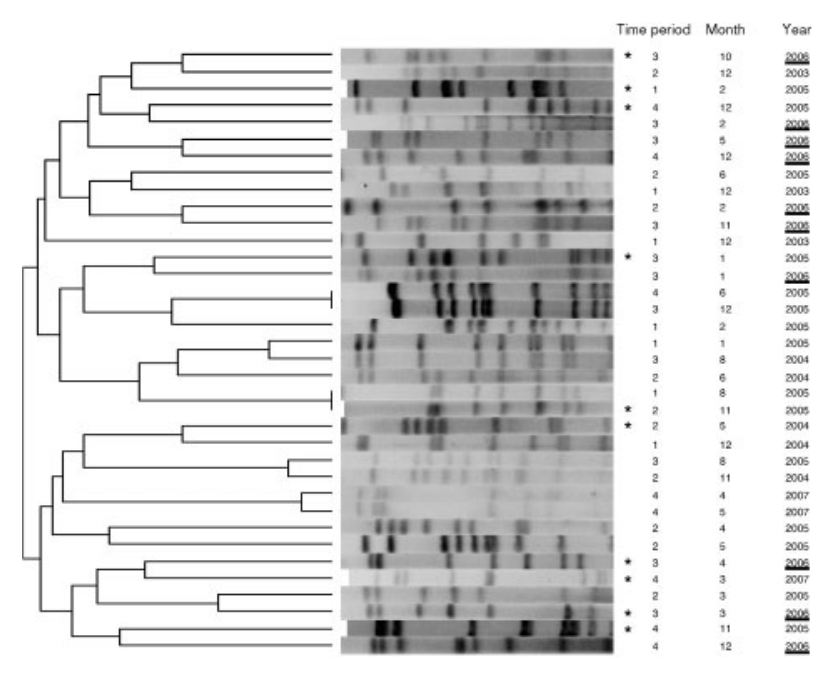

Fig. 4. Dendrogram of PFGE genotypic variation for $36 \mathrm{H}$. influenzae isolates obtained from 12 children in Rotterdam. Time period after birth when nasal swabs were taken: 1,6 weeks; 2, 6 months; 3, 14 months; 4, 24 months. The month and year of isolate culture from the nose of children are also shown (year of peak prevalence underlined). Co-colonization with $M$. catarrhalis is indicated with asterisks $\left(^{*}\right)$. Note the non-clonal nature of cocolonizing $H$. influenzae genotypes.
Surveillance Scheme (EISS), concluded that the 2005-2006 and 2006-2007 influenza seasons in Europe were characterized by moderate clinical activity (Arkema et al., 2008; Meijer et al., 2007), and as a consequence may have resulted in less viral predisposition of the middle ear epithelium to bacterial infection.

At least two studies have suggested that the presence of a particular bacterial species during URTIs may create a more hospitable niche for co-colonization by a second distinct bacterial species (Armbruster et al., 2010; Pettigrew et al., 2008). Armbruster et al. (2010) showed that $H$. influenzae promotes $M$. catarrhalis persistence within polymicrobial biofilms via inter-species quorum signalling, and that co-infection with both species promotes the increased resistance of biofilms to antibiotics and host clearance (Armbruster et al., 2010). In fact, the process of biofilm formation has been demonstrated for numerous pathogens and is clearly an important microbial survival strategy.

In our present study, co-colonization between $M$. catarrhalis and $H$. influenzae was significantly more likely than colonization with either bacterial species alone. This is an interesting result, as (for example) competition for cellular binding sites may be expected to occur in the presence of two distinct bacterial species, although this hypothesis needs further study. In detail, both species can bind to carcinoembryonic antigen-related cell adhesion molecules (CEACAM)-1, which is a receptor for the OMPs UspA1 and $\mathrm{P} 5$ of $M$. catarrhalis and $H$. influenzae, respectively

Table 2. Prevalence of virulence genes for $112 \mathrm{M}$. catarrhalis isolates for which PFGE genotyping was performed

\begin{tabular}{|lcc|}
\hline Virulence gene & Positive (\%) & Negative (\%) \\
\hline uspA1 & 97 & 3 \\
hag/mid & 80 & 20 \\
uspA2 & 83 & 17 \\
uspA2H & 17 & 83 \\
ompJ & 100 & 0 \\
\hline
\end{tabular}




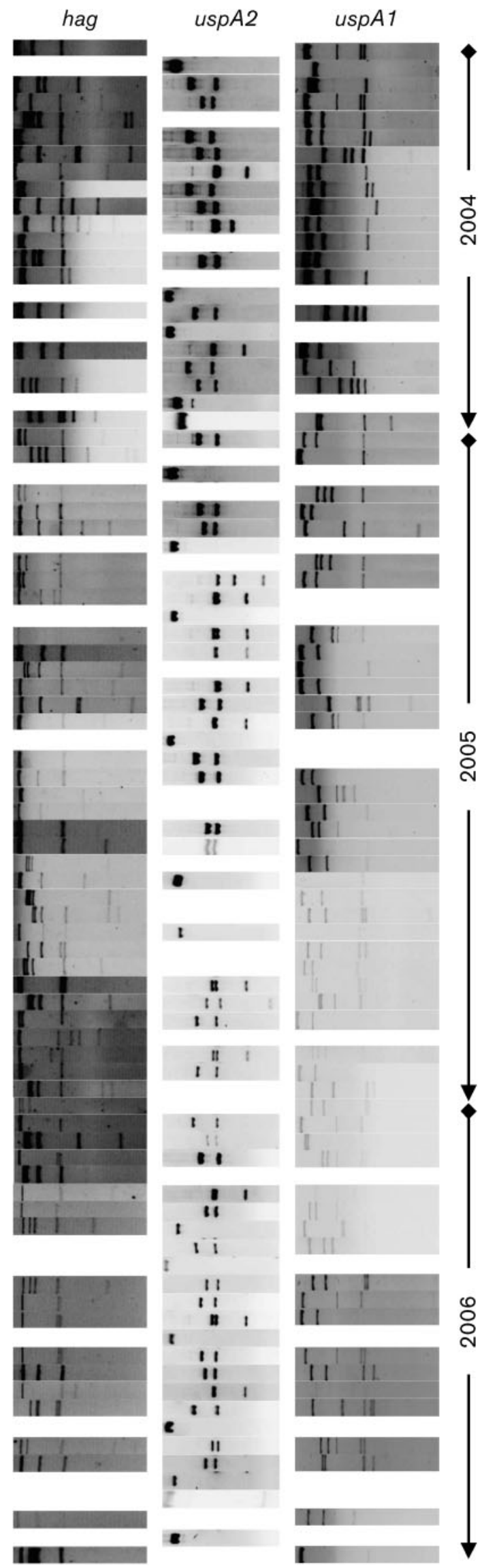

Fig. 5. Diversity of PCR-RFLP patterns obtained from the hag/ mid, uspA2 and uspA1 virulence genes of 86 colonizing $M$. catarrhalis isolates cultured during 2004-2006 from healthy children in Rotterdam, The Netherlands. Note the diversity of restriction patterns, even within the same year, and that there is no linkage between genotype pattern combinations for the three immunologically relevant virulence genes tested.

(Hill et al., 2001). CEACAM-1 is a specific innate immune receptor and although the CEACAM-binding ligands of respiratory pathogens are structurally diverse, they target a common site on the receptor (Hill et al., 2005; Sarantis \& Gray-Owen, 2007). However, competition for binding sites could be outweighed by non-receptor-mediated factors associated with co-colonization events. For example, $M$. catarrhalis releases outer-membrane vesicles (OMVs) or 'blebs' from its surface during growth in several environments, e.g. liquid culture, solid culture and biofilms. The OMVs that are secreted by $M$. catarrhalis carry UspA1 and UspA2, which are known to interfere with the activation of the complement cascade. Tan et al. (2007) showed that blebs carrying UspA1/A2 protected $H$. influenzae from complement-mediated killing, suggesting that $M$. catarrhalis may promote the survival of $H$. influenzae during cocolonization (Tan et al., 2007). Deich \& Hoyer (1982) demonstrated the generation and release of DNA-binding vesicles by $H$. influenzae (Deich \& Hoyer, 1982), although this study did not mention the effect of $H$. influenzae blebs on the complement cascade. At this time, no other studies have reported the presence of $H$. influenzae blebs and their possible beneficial effect on other bacterial species in co-culture.

Whatever the exact cause of co-colonization dominance, neither co-colonizing $M$. catarrhalis nor $H$. influenzae belonged to specific genotypic clones, suggesting that a ubiquitous system is facilitating the significant difference in co-colonization (versus single-species colonization) observed in this study. As recently described by Armbruster et al. (2010), the most likely (non-clonal-genotype-related) ubiquitous system driving co-colonization events of these two bacterial pathogens appears to be the quorum sensing system (Armbruster et al., 2010). However, complement-evading systems, e.g. via OMV-mediated protection, may also play a significant role.

Genotypic analysis revealed a high degree of diversity in colonizing $M$. catarrhalis isolates, an observation which is in agreement with previous studies showing that genotypic heterogeneity is typical of worldwide M. catarrhalis isolates colonizing both children and adults (Hays et al., 2003; Verhaegh et al., 2008; Wolf et al., 2000). Also, no significant association between genotype (cluster) and season, or year of isolation was observed, which is in contrast with a previous publication by Levy et al. (2009) who reported that winter and spring season was a significant risk factor for clustering. However, Levy et al. (2009) studied transmission between patients within a 
relatively confined hospital setting (Levy et al., 2009), whereas the focus of our study was related to community transmission.

The prevalence of the virulence genes uspA1, uspA2 and hag/mid within our Rotterdam isolates was very similar to that previously reported for global M. catarrhalis isolates (Verhaegh et al., 2008). These virulence genes encode OMPs that play an important role in bacterial adherence to human epithelia and complement resistance, as well as being immunogenic (Helminen et al., 1994, 1995; Meier et al., 2003). This finding suggests that equilibrium has been reached with respect to virulence gene distribution in global M. catarrhalis isolates, as local geographical factors do not appear to generate significant differences in virulence gene prevalence.

Finally, a high degree of gene sequence diversity was identified in uspA1, uspA2 and hag/mid virulence genes during the 3-year study period, with no apparent linkage between uspA1, uspA2 and hag/mid banding patterns. Moreover, the PCR-RFLP method used was chosen to target restriction sites associated with immunologically important regions of the corresponding UspA1, UspA2 and Hag/MID proteins. The lack of intra-gene band pattern clustering and lack of inter-gene band pattern linkage indicate that these three immunologically relevant virulence genes may be experiencing immune pressure, each separately adapting to evade the human immune response. The consequence would be immune evasion and an increased ability to colonize human populations over time.

\section{Conclusion}

Regular 6-monthly cycles of $M$. catarrhalis and $H$. influenzae colonization peaks were observed throughout 2004-2006 in healthy children residing in Rotterdam, The Netherlands. Colonization was characterized by a high degree of $M$. catarrhalis genotypic diversity that was maintained over the 3-year study period, and was independent of year and season of isolation. Genetic diversity was observed in three immunologically important virulence genes, with no clustering of band pattern types during particular months or years. This high degree of whole-cell genotypic variation, coupled to independent (unlinked) virulence gene variation, is likely to be one of the factors ensuring the success of $M$. catarrhalis colonization events in the early years of life, facilitating immune evasion and further cycles of colonization.

The fact that the co-colonization prevalence was significantly greater than single-species prevalence alone for these two microbial pathogens adds weight to the recently published findings indicating the importance of quorumsensing-mediated inter-species cooperation in biofilm maturation and increased resistance to host clearance. Our study provides the first evidence to indicate the importance of this quorum-sensing interaction within the community setting.

\section{ACKNOWLEDGEMENTS}

The authors gratefully acknowledge the contribution of general practitioners, hospitals, midwives and pharmacies in Rotterdam, including the Erasmus Medical Center (MC), Rotterdam, the School of Law and Faculty of Social Sciences of the Erasmus University Rotterdam, the Municipal Health Service Rotterdam area, the Rotterdam Homecare Foundation and the Stichting Trombosedienst \& Artsenlaboratorium Rijnmond (STAR), Rotterdam, who are conducting the Generation R Focus Cohort Study. We would also like to acknowledge Ad Luijendijk for technical supervision at the Department of Medical Microbiology and Infectious Diseases, Erasmus MC, Rotterdam. The first phase of the Generation R Study was made possible by financial support from the Erasmus MC, Rotterdam, the Erasmus University Rotterdam and The Netherlands Organization for Health Research and Development (ZonMw). Additionally, an unrestricted grant from the ECT Rotterdam funded this project. Finally, this work was supported by a grant from the European Union, FP6 Project OMVac 037653. All authors declare that they have no conflicts of interest.

\section{REFERENCES}

Akimana, C. \& Lafontaine, E. R. (2007). The Moraxella catarrhalis outer membrane protein CD contains two distinct domains specifying adherence to human lung cells. FEMS Microbiol Lett 271, 12-19.

Arkema, J. M., Meijer, A., Meerhoff, T. J., Van Der Velden, J. \& Paget, W. J. (2008). Epidemiological and virological assessment of influenza activity in Europe, during the 2006-2007 winter. Euro Surveill 13, 18958.

Armbruster, C. E., Hong, W., Pang, B., Weimer, K. E., Juneau, R. A., Turner, J. \& Swords, W. E. (2010). Indirect pathogenicity of Haemophilus influenzae and Moraxella catarrhalis in polymicrobial otitis media occurs via interspecies quorum signaling. MBio 1, e00102-e00110.

Bakaletz, L. O. (1995). Viral potentiation of bacterial superinfection of the respiratory tract. Trends Microbiol 3, 110-114.

Balder, R., Hassel, J., Lipski, S. \& Lafontaine, E. R. (2007). Moraxella catarrhalis strain O35E expresses two filamentous hemagglutinin-like proteins that mediate adherence to human epithelial cells. Infect Immun 75, 2765-2775.

Berner, R., Schumacher, R. F., Brandis, M. \& Forster, J. (1996). Colonization and infection with Moraxella catarrhalis in childhood. Eur J Clin Microbiol Infect Dis 15, 506-509.

Bullard, B., Lipski, S. L. \& Lafontaine, E. R. (2005). Hag directly mediates the adherence of Moraxella catarrhalis to human middle ear cells. Infect Immun 73, 5127-5136.

Deich, R. A. \& Hoyer, L. C. (1982). Generation and release of DNAbinding vesicles by Haemophilus influenzae during induction and loss of competence. J Bacteriol 152, 855-864.

Edwards, K. J., Schwingel, J. M., Datta, A. K. \& Campagnari, A. A. (2005). Multiplex PCR assay that identifies the major lipooligosaccharide serotype expressed by Moraxella catarrhalis clinical isolates. J Clin Microbiol 43, 6139-6143.

Faden, H., Waz, M. J., Bernstein, J. M., Brodsky, L., Stanievich, J. \& Ogra, P. L. (1991). Nasopharyngeal flora in the first three years of life in normal and otitis-prone children. Ann Otol Rhinol Laryngol 100, 612-615.

Faden, H., Duffy, L., Wasielewski, R., Wolf, J., Krystofik, D. \& Tung, Y. (1997). Relationship between nasopharyngeal colonization and the development of otitis media in children. Tonawanda/Williamsville pediatrics. J Infect Dis 175, 1440-1445. 
Forsgren, A., Brant, M., Mollenkvist, A., Muyombwe, A., Janson, H., Woin, N. \& Riesbeck, K. (2001). Isolation and characterization of a novel IgD-binding protein from Moraxella catarrhalis. J Immunol 167, 2112-2120.

Freid, V. M., Makuc, D. M. \& Rooks, R. N. (1998). Ambulatory health care visits by children: principal diagnosis and place of visit. Vital Health Stat 13, 1-23.

Gonzales, R., Malone, D. C., Maselli, J. H. \& Sande, M. A. (2001). Excessive antibiotic use for acute respiratory infections in the United States. Clin Infect Dis 33, 757-762.

Hays, J. P., Eadie, K., Verduin, C. M., Hazelzet, J., Verbrugh, H. \& van Belkum, A. (2003). Changes in genetic types and population dynamics of Moraxella catarrhalis in hospitalized children are not associated with an exacerbation of existing disease. $J$ Med Microbiol 52, 815-820.

Helminen, M. E., Maciver, I., Latimer, J. L., Klesney-Tait, J., Cope, L. D., Paris, M., McCracken, G. H., Jr \& Hansen, E. J. (1994). A large, antigenically conserved protein on the surface of Moraxella catarrhalis is a target for protective antibodies. J Infect Dis 170, 867-872.

Helminen, M. E., Beach, R., Maciver, I., Jarosik, G., Hansen, E. J. \& Leinonen, M. (1995). Human immune response against outer membrane proteins of Moraxella (Branhamella) catarrhalis determined by immunoblotting and enzyme immunoassay. Clin Diagn Lab Immunol 2, 35-39.

Hendley, J. O., Hayden, F. G. \& Winther, B. (2005). Weekly point prevalence of Streptococcus pneumoniae, Haemophilus influenzae and Moraxella catarrhalis in the upper airways of normal young children: effect of respiratory illness and season. APMIS 113, 213-220.

Hill, D. J. \& Virji, M. (2003). A novel cell-binding mechanism of Moraxella catarrhalis ubiquitous surface protein UspA: specific targeting of the $\mathrm{N}$-domain of carcinoembryonic antigen-related cell adhesion molecules by UspA1. Mol Microbiol 48, 117-129.

Hill, D. J., Toleman, M. A., Evans, D. J., Villullas, S., Van Alphen, L. \& Virji, M. (2001). The variable P5 proteins of typeable and non-typeable Haemophilus influenzae target human CEACAM1. Mol Microbiol 39, 850-862.

Hill, D. J., Edwards, A. M., Rowe, H. A. \& Virji, M. (2005). Carcinoembryonic antigen-related cell adhesion molecule (CEACAM)-binding recombinant polypeptide confers protection against infection by respiratory and urogenital pathogens. Mol Microbiol 55, 1515-1527.

Jaddoe, V. W., Bakker, R., van Duijn, C. M., van der Heijden, A. J., Lindemans, J., Mackenbach, J. P., Moll, H. A., Steegers, E. A., Tiemeier, H. \& other authors (2007). The Generation R Study Biobank: a resource for epidemiological studies in children and their parents. Eur J Epidemiol 22, 917-923.

Jaddoe, V. W., van Duijn, C. M., van der Heijden, A. J., Mackenbach, J. P., Moll, H. A., Steegers, E. A., Tiemeier, H., Uitterlinden, A. G., Verhulst, F. C. \& Hofman, A. (2008). The Generation R Study: design and cohort update until the age of 4 years. Eur J Epidemiol 23, 801-811.

Konno, M., Baba, S., Mikawa, H., Hara, K., Matsumoto, F., Kaga, K., Nishimura, T., Kobayashi, T., Furuya, N. \& other authors (2006). Study of upper respiratory tract bacterial flora: first report. Variations in upper respiratory tract bacterial flora in patients with acute upper respiratory tract infection and healthy subjects and variations by subject age. J Infect Chemother 12, 83-96.

Leibovitz, E. (2003). Acute otitis media in pediatric medicine: current issues in epidemiology, diagnosis, and management. Paediatr Drugs $\mathbf{5}$ (Suppl. 1), 1-12.

Levy, F., Leman, S. C., Sarubbi, F. A. \& Walker, E. S. (2009). Nosocomial transmission clusters and risk factors in Moraxella catarrhalis. Epidemiol Infect 137, 581-590.
Lipski, S. L., Akimana, C., Timpe, J. M., Wooten, R. M. \& Lafontaine, E. R. (2007). The Moraxella catarrhalis autotransporter $\mathrm{McaP}$ is a conserved surface protein that mediates adherence to human epithelial cells through its $\mathrm{N}$-terminal passenger domain. Infect Immun 75, 314-324.

Liu, D. F., McMichael, J. C. \& Baker, S. M. (2007). Moraxella catarrhalis outer membrane protein $\mathrm{CD}$ elicits antibodies that inhibit CD binding to human mucin and enhance pulmonary clearance of $M$. catarrhalis in a mouse model. Infect Immun 75, 2818-2825.

Marchisio, P., Gironi, S., Esposito, S., Schito, G. C., Mannelli, S. \& Principi, N. (2001). Seasonal variations in nasopharyngeal carriage of respiratory pathogens in healthy Italian children attending day-care centres or schools. J Med Microbiol 50, 1095-1099.

Meier, P. S., Freiburghaus, S., Martin, A., Heiniger, N., Troller, R. \& Aebi, C. (2003). Mucosal immune response to specific outer membrane proteins of Moraxella catarrhalis in young children. Pediatr Infect Dis J 22, 256-262.

Meijer, A., Meerhoff, T. J., Meuwissen, L. E., van der Velden, J. \& Paget, W. J. (2007). Epidemiological and virological assessment of influenza activity in Europe during the winter 2005-2006. Euro Surveill 12, E11-E12.

Moor, P. E., Collignon, P. C. \& Gilbert, G. L. (1999). Pulsed-field gel electrophoresis used to investigate genetic diversity of Haemophilus influenzae type $\mathrm{b}$ isolates in Australia shows differences between Aboriginal and non-Aboriginal isolates. J Clin Microbiol 37, 15241531.

Murphy, T. F. \& Parameswaran, G. I. (2009). Moraxella catarrhalis, a human respiratory tract pathogen. Clin Infect Dis 49, 124-131.

Peerbooms, P. G., Engelen, M. N., Stokman, D. A., van Benthem, B. H., van Weert, M. L., Bruisten, S. M., van Belkum, A. \& Coutinho, R. A. (2002). Nasopharyngeal carriage of potential bacterial pathogens related to day care attendance, with special reference to the molecular epidemiology of Haemophilus influenzae. J Clin Microbiol 40, 28322836.

Pettigrew, M. M., Gent, J. F., Revai, K., Patel, J. A. \& Chonmaitree, T. (2008). Microbial interactions during upper respiratory tract infections. Emerg Infect Dis 14, 1584-1591.

Pichichero, M. E. (2000). Short course antibiotic therapy for respiratory infections: a review of the evidence. Pediatr Infect Dis $J$ 19, 929-937.

Plasschaert, A. I., Rovers, M. M., Schilder, A. G., Verheij, T. J. \& Hak, E. (2006). Trends in doctor consultations, antibiotic prescription, and specialist referrals for otitis media in children: 1995-2003. Pediatrics 117, 1879-1886.

Principi, N., Marchisio, P., Schito, G. C. \& Mannelli, S. (1999). Risk factors for carriage of respiratory pathogens in the nasopharynx of healthy children. Ascanius Project Collaborative Group. Pediatr Infect Dis J 18, 517-523.

Riesbeck, K., Tan, T. T. \& Forsgren, A. (2006). MID and UspA1/A2 of the human respiratory pathogen Moraxella catarrhalis, and interactions with the human host as basis for vaccine development. Acta Biochim Pol 53, 445-456.

Ruckdeschel, E. A., Kirkham, C., Lesse, A. J., Hu, Z. \& Murphy, T. F. (2008). Mining the Moraxella catarrhalis genome: identification of potential vaccine antigens expressed during human infection. Infect Immun 76, 1599-1607.

Ruckdeschel, E. A., Brauer, A. L., Johnson, A. \& Murphy, T. F. (2009). Characterization of proteins Msp22 and Msp75 as vaccine antigens of Moraxella catarrhalis. Vaccine 27, 7065-7072.

Ruuskanen, O., Arola, M., Heikkinen, T. \& Ziegler, T. (1991). Viruses in acute otitis media: increasing evidence for clinical significance. Pediatr Infect Dis J 10, 425-427. 
Sarantis, H. \& Gray-Owen, S. D. (2007). The specific innate immune receptor CEACAM3 triggers neutrophil bactericidal activities via a Syk kinase-dependent pathway. Cell Microbiol 9, 2167-2180.

Slevogt, H., Zabel, S., Opitz, B., Hocke, A., Eitel, J., N'guessan, P. D., Lucka, L., Riesbeck, K., Zimmermann, W. \& other authors (2008). CEACAM1 inhibits Toll-like receptor 2-triggered antibacterial responses of human pulmonary epithelial cells. Nat Immunol 9, $1270-1278$.

Tan, T. T., Christensen, J. J., Dziegiel, M. H., Forsgren, A. \& Riesbeck, K. (2006a). Comparison of the serological responses to Moraxella catarrhalis immunoglobulin D-binding outer membrane protein and the ubiquitous surface proteins A1 and A2. Infect Immun 74, 63776386.

Tan, T. T., Forsgren, A. \& Riesbeck, K. (2006b). The respiratory pathogen Moraxella catarrhalis binds to laminin via ubiquitous surface proteins A1 and A2. J Infect Dis 194, 493-497.

Tan, T. T., Morgelin, M., Forsgren, A. \& Riesbeck, K. (2007). Haemophilus influenzae survival during complement-mediated attacks is promoted by Moraxella catarrhalis outer membrane vesicles. $J$ Infect Dis 195, 1661-1670.

Verduin, C. M., Kools-Sijmons, M., van der Plas, J., Vlooswijk, J., Tromp, M., van Dijk, H., Banks, J., Verbrugh, H. \& van Belkum, A. (2000). Complement-resistant Moraxella catarrhalis forms a genetically distinct lineage within the species. FEMS Microbiol Lett 184, 1-8.
Verduin, C. M., Hol, C., Fleer, A., van Dijk, H. \& van Belkum, A. (2002). Moraxella catarrhalis: from emerging to established pathogen. Clin Microbiol Rev 15, 125-144.

Verhaegh, S. J., Streefland, A., Dewnarain, J. K., Farrell, D. J., van Belkum, A. \& Hays, J. P. (2008). Age-related genotypic and phenotypic differences in Moraxella catarrhalis isolates from children and adults presenting with respiratory disease in 2001-2002. Microbiology 154, 1178-1184.

Verhaegh, S. J., Lebon, A., Saarloos, J. A., Verbrugh, H. A., Jaddoe, V. W., Hofman, A., Hays, J. P., Moll, H. A. \& van Belkum, A. (2010). Determinants of Moraxella catarrhalis colonization in healthy Dutch children during the first 14 months of life. Clin Microbiol Infect 16, 992-997.

Vlastarakos, P. V., Nikolopoulos, T. P., Korres, S., Tavoulari, E., Tzagaroulakis, A. \& Ferekidis, E. (2007). Grommets in otitis media with effusion: the most frequent operation in children. But is it associated with significant complications? Eur J Pediatr 166, 385-391.

Wolf, B., Kools-Sijmons, M., Verduin, C., Rey, L. C., Gama, A., Roord, J., Verhoef, J. \& van Belkum, A. (2000). Genetic diversity among strains of Moraxella catarrhalis cultured from the nasopharynx of young and healthy Brazilian, Angolan and Dutch children. Eur J Clin Microbiol Infect Dis 19, 759-764.

Edited by: P. van der Ley 